\title{
Clustering Schemes Use for To Mobile Ad Hoc Network
}

\author{
P. A. Gaikwad*, Dr. S. S. Sherekar, Dr. V. M. Thakare
}

PG Department of Computer Science and Engineering, Sant Gadge Baba Amravati University, Amravati, Maharashtra, India

Article Info

Volume 7, Issue 2

Page Number: 137-142

Publication Issue :

March-April-2021

\section{Article History}

Accepted : 01 March 2021

Published : 08 March 2021

\section{ABSTRACT}

The MANETs are effectively deployed in the different environments such as: Armed Forces, Disaster Management, Medical and other similar Integrated Applications . Observed that new challenges and applications come up in the MANETs in every short period of a time. In the large deployments MANETs can have huge number of mobile nodes creating new problems. In the order to overcome such challenges, clustering algorithms are a invariably used to allow the structuring of the network Clustering methods allow fast connection and also for better routing and to the topology management of mobile ad hoc networks (MANET). The assortment of approaches for the MANET grouping has been created by scientists which center around various kinds of execution measurements. In this paper presents a analysis of various clustering schemes for the MANET into group of entities or the cluster and proposes efficient approach.

Keywords : - Ad Hoc Network MANETS.

\section{INTRODUCTION}

In all the nodes are routers connected with wireless network. The switches can allowed to move and coordinate themselves self-assertively, at that point an organization geography may change quickly and an unusually. In that an organization may work in an independent way, or might be associated with the bigger Internet or might be utilized as the mixture remote network[1]. It is unique characteristics of MANETs result in the research challenges due to the limited bandwidth and the frequent link rate variations. Particular limited bandwidth is caused by the wireless links between mobile devices[2]. In This bandwidth is much lower than that provided by wired links. The MANETs are formed by group of nodes that can be transmit and a receive data and also a relay data among themselves. The communication between nodes is made over the wireless links[3]. Such a pair of nodes can establish a wireless link among themselves only if they are within transmission range of the each other. Main node is a mobile host connected wirelessly to other nodes[4]. When communicating transmission from such devices is received by all other a nodes that lie within a transmission range in that, if both sender \& receiver nodes are out of range from each other, in the MANET, then a intermediate mobile nodes have the ability to construct a communication path effectively and then and then only after forward the packets usually under the supervision of Cluster Head (CHO wing to its nature of mobility, each host has a

Copyright: () the author(s), publisher and licensee Technoscience Academy. This is an open-access article distributed under the terms of the Creative Commons Attribution Non-Commercial License, which permits unrestricted non-commercial use, distribution, and reproduction in any medium, provided the original work is properly cited 
function independently. At that point the host is fit for moving anyplace arbitrarily and needs to adjust to its dynamic geography. An Application of such technology is witnessed in educational, military and industrial sector etc, [5]

\section{BACKGROUND}

The MANETs are formed by the groups of nodes that can be transmit and a receive data and also a relay data among them. The communication between nodes are made over wireless links. The pair of nodes can establish wireless link among themselves only when they are within transmission range of each other. The important feature of the ad hoc networks is that routes between the two hosts may be consist of a hops through other hosts in a network. When the sender send node wants to the communicate with the receiver node If it may happen that they are not within a communication range from each other. In this paper, analysis of many existing cluster.

The paper is organized as follows. Section I Introduction. Section II talks about the Background. Section III explains previous work done before on clustering schemes use for to mobile ad hoc network. Section IV gives a brief about existing methodologies. Section V gives the proposed method Section VI gives the Pros. Section VII gives the Cons. Section VIII Conclude this paper. Finally, Section IX gives Result.

\section{PREVIOUS WORK DONE}

Umamaheswari et.al (2012) [1] proposed that present the review of the different clustering techniques and the large benefits that come along with when clustering approaches are implemented in the MANET.

Sandeep Monga et.al (2019) [2] proposed that analysis of many existing clustering algorithms of
MANETs to classify such as: Identity based, Mobility based, Topology based, Energy based, Weight based and artificial intelligence based clustering is done.

Yuanzhu Peter Chen et. al (2004) [3] proposed that survey several clustering algorithms, concentrating on those that are based on graph domination.

\section{EXISTING METHODOLOGY}

In routing scheme in the dynamic environment such as ad hoc networks must consider that a topology of a network can be vary while a packet is being routed and in that the quality of a wireless links is highly variable. In the wired networks, link failure is does not a frequent since in the network structure is mostly static[1]. Flooding is used for the MANET to propagate control messages. Flooding is the distributed process . which is the node transmits a message to all its a neighbours and so transmit the message consecutively to the neighbours and goes on until the message has been disseminated to the entire network. Owing to the its nature of mobility, each host has to a function independently[2]. A host is capable for moving anywhere randomly and has to a adapt to its dynamic topology. Application of such technology is witnessed in the educational, military and industrial sector networks flat structure topology is the quite in effective as it is a quite complicated and unsteady due to node mobility. Looking forward to other option, a researchers have proposed hierarchical topology structure, to overcome a above stated problem. In this approach, node is notionally put in the group called Cluster. Every other node maintains whole information of its a cluster and the part information of other clusters, resulting is to improved routing efficiency of the dynamic network[4]. 


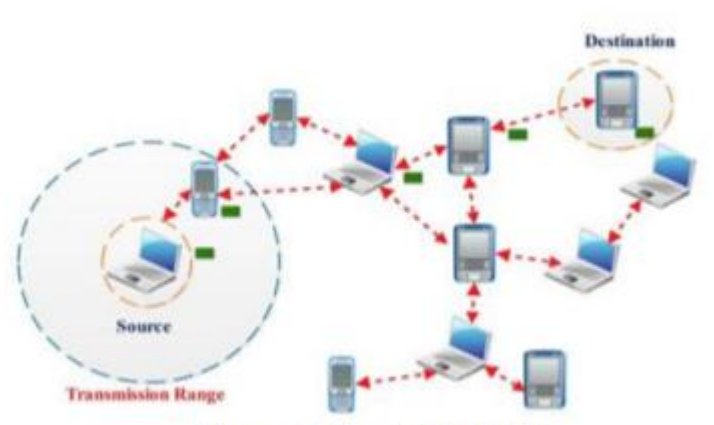

Figure-1 Simple MANET

Node $\mathrm{A}$ and $\mathrm{C}$ are not In MANET creation of the Cluster refers to forming groups of nodes at in the beginning in a structure called as cluster for organizing wireless network[5]. The cluster regularly updates a route tables and a occasionally reselects the $\mathrm{CH}$ in a network. At all the times with a cluster mobile nodes must be a aware of any neighbourhood node changes. Then and then only cluster maintenance necessitates of frequent updating the clusters and cluster heads is direct transmission range. .Cluster-Head $(\mathrm{CH})$ : One of the nodes in the every cluster can chosen as $\mathrm{CH}$ which a monitors and the controls communication routes for all other a nodes with its own domain. Cluster -Gateway (CG): In this type of a node exists in more than one cluster and act as the bridge between $\mathrm{CH}$ of that clusters. The cluster Head and the Cluster Gateway nodes are the backbone of the network. Normal Node: This type of the node sends the information to the $\mathrm{CH}$ which can be turn forwards the gathered information to the next hop.

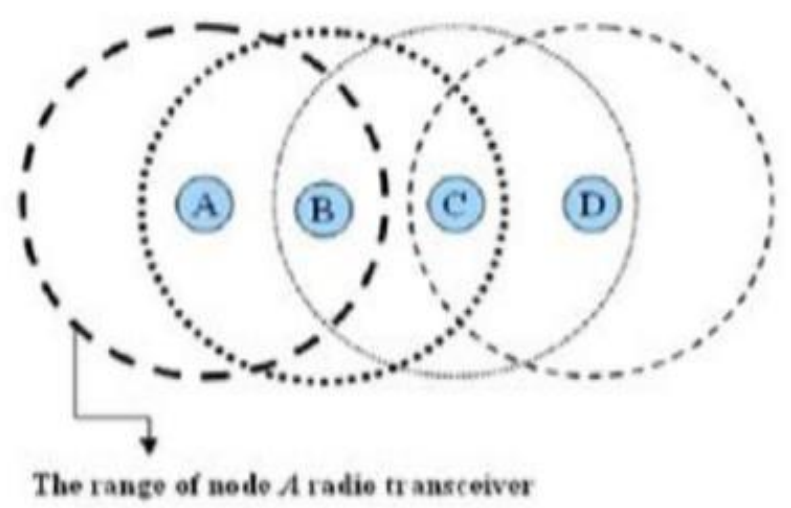

Figure-2 Simple Network Model for MANETS

\section{PROPOSED METHODOLOGY}

In the research of many authors standard protocols and their abilities, have been evaluated assuming that deployment configurations such as varying degrees of a node mobility within the limited space, implying that all over nodes lie within the few hops of each other. In the performance evaluation of protocols, parameters namely a packet drop rate, end-to-end packet delays, network throughput, the overhead introduced by the routing protocol, etc. can be used. The limited bandwidth ad-hoc network makes to imperative to construct a the virtual network with a subset of the nodes and there from identifying a route for forwarding the packets for good puts. Nodes belonging to neighbouring clusters communicate with boundary nodes. In the gathering the hubs into unmistakable groups for structure appropriate bunches is one of the problem area and greatest difficulties MANET research territories. Optimal clustering solutions can be greatly enhance to the practicability and the performance of MANETs. In direct node to node communication happen amongst those a nodes which are within the radio range of each other. The outside of a radio range nodes communicate with a assistance of the intermediate nodes to a route their packets. So that MANETs can work an any place in any topography. This can gives the distinctive edge to the MANET and makes it highly robust. Nodes $\mathrm{A}$ and $\mathrm{C}$ in Figure-2 must be trace the route through node $B$ to a communicate with each other. The nominal radio range of a each node is depicted by the circles. 


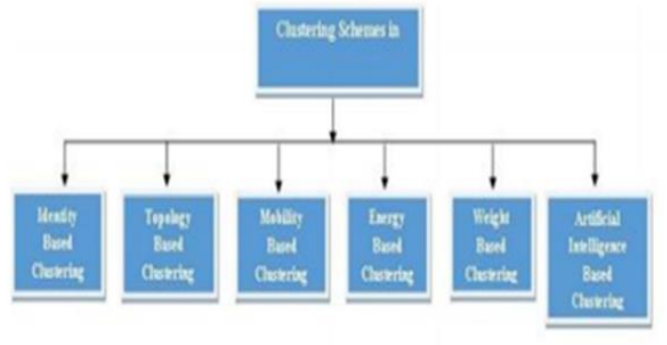

Figure-4 Various Clustering Schemes in MANET

Identity Based Clustering:

(A) Lowest ID cluster algorithm (LIC) :

The node with the minimum id is chosen as the cluster head in a Lowest ID cluster algorithm (LIC) in which is a neighbours of a cluster head will having a higher ID than that of a cluster head. The node which are lies within a transmission range of two or more cluster heads will acting as the gateway a node which is used for the routing between clusters. Each of anode is assigned to the distinct id. A Lowest-ID scheme concerns only with a lowest node ids which are arbitrarily assigned numbers without Since a node ids do not change within a time, those with the smaller ids is more likely to become a cluster heads than a nodes with larger ids. Thus, drawback of the lowest ID algorithm is that certain nodes is prone to the power drainage due to this serving as cluster heads for a longer periods of a time. In this paper, the clustering algorithm known as LCA (Linked Cluster Algorithm) are proposed where every a node is a $\mathrm{CH}$, either the Gateway Node or a Normal Node. At first stage, all the nodes will be normal node. In the preset time interval, every node is in a network will be broadcast its ID to its neighbour nodes. In the node with a lowest Id is elected as a CH.[7]

(B) Weighted clustering Algorithm(WCA):

The distributed clustering algorithm based on a weight values is proposed to the weighted clustering algorithm (WCA) selects cluster heads. Therefore, in the order to optimize battery usage, load balancing and MAC functionality the node is chosen to be the cluster head according to a number of nodes it can behandle, mobility, transmission power and battery power, etc. WCA selects a cluster heads according to a weight value of the each node. A weight associated to the node $\mathrm{v}$ is defined as $\mathrm{Wv}=\mathrm{w} 1 \_\mathrm{v}+\mathrm{w} 2 \mathrm{Dv}+$ $\mathrm{w} 3 \mathrm{Mv}+\mathrm{w} 4 \mathrm{Pv}$ a node with a minimum weight is selected as a cluster head. In the weighting factors is chosen so that $\mathrm{w} 1+\mathrm{w} 2+\mathrm{w} 3+\mathrm{w} 4=1$. A cluster head selection algorithm finishes once all anodes become either the cluster head or the member of a cluster head. The distance between the members of a cluster head, must be a less or equal to a transmission range between them. No two cluster heads are immediate neighbours.[8]

(C) The Least Cluster Change algorithm ( LCC) :

In this put forth, wherein the algorithm maintenance steps is added to a optimize in the re-clustering cost. A re-formation of the cluster is done only in a two conditions: first, when a two cluster heads is neighbour node, a node with utmost id will be selected as $\mathrm{CH}$. Second, if the member node moves in to a another cluster then a node leaving a cluster will be the $\mathrm{CH}$. And the process to select a new cluster will be initiated.

\section{(D) Topology Based Clustering:}

In the paper, authors have proposed the clustering based new link state routing algorithm is called CLSR for the MANET. It is a claimed that the size of a routing table and routing overhead are deduced. In the redundant information on different control a packet types is eliminated by this algorithm and traffic control is a reduced. Two types of the control messages HELLO and CTC is used in CLSR. For the clustering process each node send - Helloll message and a responses are used for the computation of the 2-hops local routes. The $\mathrm{CH}$ initiates CTC message 
which are broadcast throughout a whole network. This is allows a nodes to compute inter-cluster routes. For the simulation result shows the best performance of this algorithm in the terms of a routing overhead and a data packet delivery ratio.[10]

(E) Max-min d-cluster :

In the most of a clustering algorithms, a cluster head election is done in such a way that no node can be more than the one hop away from its cluster head. A main drawback suggested by of nodes being 1-hop away from the cluster head, it is that generates a large number of the cluster heads within a network causing the congestion problem. Therefore, in a MaxMin heuristic clusters is formed by anodes that can be the at most d-hops away from a cluster head. The d-neighborhood of the hub comprises of a hub itself and afterward set of the relative multitude of hubs situated inside $\mathrm{d}$-jumps from a hub. In this heuristic $\mathrm{d}$ is defined as a maximum number of the hops away from a nearest cluster head $\left(\mathrm{d}_{-} 1\right)$. This value is the input to a heuristic allowing control over a number of the cluster heads to be selected. The node determines it as a gateway node if it has a neighbouring nodes with the different cluster heads. After the node has identified that it is gateway node it sends to SENDER array. In this manner the cluster head can be obtain information of the every node in its cluster. This heuristic maximizes a number of the gateways in order to obtain the backbone formed by multiple paths between neighbouring cluster heads. In this way, congestion is eased.[9]

\section{(F) Mobility Based Clustering:}

The new mobility metric for anodes in the Mobile Ad -hoc Networks. It is easy to calculate and does not require an any knowledge of the fixed locations. The proposed mobility based clustering algorithm called MOBIC . Based on the simulation results, they are deduced that relative mobility is better parameter for clustering anodes rather than a plain IDs which is not representative of a node mobility in MANETs. For network with high mobility nodes, authors have proposed the Mobility prediction-based clustering algorithm (MPBC). The proposed algorithm has two a phases initial clustering phase and a cluster maintenance phase. In this algorithm depends on a estimates about mobility information. Thereafter its working becomes independent of external a device (e.g. GPS). Simulation results show effective performance in the terms of a network lifetime, reduced reaffiliation rate and the re-association time.[11]

\section{(G) Energy Based Clustering:}

The node battery energy has the direct effect on the network lifetime. In the energy limitation puts up the severe network performance challenge In this paper, researchers had proposed a algorithm called —Flexible weighted Clustering Algorithm based on the Battery Powerl (FWCABP) [25]. This is the algorithm functional only when a demand is present due to the node movement resulting in changes the relative distance between a cluster-head and the node. Weights are assigned to the node based on a values of some. Maintenance and the selection of $\mathrm{CH}$ are done based on a basis of information about neighbouring a nodes. The role of ordinary node and $\mathrm{CH}$ is switched to a check battery power consumption.[12]

\section{PROS:}

1)It is decreases scale of the routing tables, and an enhances the routing.

2)Updating is routing tables due to topological change results in the reduction of transmission overheads.

3)It can helps to reduce a bandwidth requirement and a energy utilization in ad-hoc networks. 


\section{CONS}

1)The communication Complexity increases due to control message exchange.

2)There is a no general solution for the clustering.

\section{CONCLUSION}

In this paper a review of several clustering schemes which help organize mobile ad hoc networks in the hierarchical manner to enable hybrid routing has been done. Clustering methods improve a network scalability, routing and topology management of the MANET.

Max-min d-cluster algorithms use flooding for a selecting the cluster head which increases a communication overhead. In the covered a basic concepts about clustering, its definition, cost, objectives \& goals of clustering schemes along with pros \& cons of clustering. After that a tried to classify the clustering schemes into many categories based on their features and objectives as: Identity based clustering, Topology based clustering, Mobility based clustering, and Artificial Intelligence based clustering.

\section{RESULT}

In this paper draws analysis of the different clustering schemes and the provides suggestions for improvement. Bunching is a cycle a recognizing some of the hubs to be assembled and a related group head. This is process plays the indispensable role in an enhancing network performance and resource management.

\section{ACKNOWLEDGMENT}

It gives immense pleasure to express my gratitude to Dr. V. M. Thakare, my guide who provided me constructive criticism and positive feedback during the preparation of this paper. I am indebted to Dr. V.
M. Thakare, Head of P. G. Department of computer science and engineering at SGBAU, Amravati, and other teaching and non-teaching staff who were always there whenever I needed any help. Without them and their co-operation, completion of this research work would have been inevitable ad their presence behind me is indispensable.

\section{REFERENCES}

[1]. H. Yan, J. Li, G. Sun, and H. Chen, "An optimistic power control MAC protocol for mobile ad hoc networks," in Proc. IEEE ICC, 2006, pp. 3615-3620.

[2]. Z. Haas and J. Deng, "Dual busy tone multiple access (DBTMA) - a multiple access control scheme for ad hoc networks," IEEE Trans.Comput., vol. 50, no. 6, pp. 975-985, Jun. 2002.

[3]. T. Korakis, Z. Tao, Yevgeniy Slutskiy, S.Panwar, "A Cooperative MAC protocol for Ad Hoc Wireless Networks", in Proceedings of the Fifth Annual IEEE International Conference on Pervasive Computing and Communications Workshops(PerComW'07), 2007

[4]. A. Boukerche, K. El-Khatib, L. Xu, and L. Korba, "An efficient secure distributed anonymous routing protocol for mobile and wireless ad hoc networks," Comput. Commun., vol. 28, pp. 1193- 1203, Jun. 2005.

[5]. I. D. Chakeres and E. M. Belding-Royer, "AODV routing protocol implementation design," in Proc. 24th Int. Conf. Distributed Computing Systems Workshops (ICDCSW’04), 2004, pp. 698- 703.

\section{Cite this article as :}

P. A. Gaikwad, Dr. S. S. Sherekar, Dr. V. M. Thakare, "Clustering Schemes Use for To Mobile Ad Hoc Network", International Journal of Scientific Research in Computer Science, Engineering and Information Technology (IJSRCSEIT), ISSN : 24563307, Volume 7 Issue 2, pp. 137-142, March-April 2021. Available at

doi : https://doi.org/10.32628/CSEIT217236

Journal URL : https://ijsrcseit.com/CSEIT217236 